\title{
Disentangling the stellar populations of the counter-rotating stellar disc in NGC 5719
}

\section{Morelli ${ }^{1,2}$, L. Coccato ${ }^{3}$, E. M. Corsini ${ }^{1,2}$, E. Dalla Bontà ${ }^{1,2}$, F. Bertola ${ }^{1,2}$, D. Vergani ${ }^{4}$ and L. Buson ${ }^{2}$}

\author{
${ }^{1}$ Dipartimento di Fisica e Astronomia G. Galilei, Università di Padova, vicolo dell'Osservatorio \\ 3, I-35122 Padova, Italy \\ email: lorenzo.morelli@unipd.it \\ ${ }^{2}$ INAF-Osservatorio Astronomico di Padova, vicolo dell'Osservatorio 2, I-35122 Padova, Italy \\ ${ }^{3}$ European Southern Observatory, Karl-Schwarzschild-Straße 2, D-85748 Garching bei \\ München, Germany \\ ${ }^{4}$ INAF, Osservatorio Astronomico di Bologna, via Ranzani 1, I-40127 Bologna, Italy
}

\begin{abstract}
We present the stellar populations properties of the interacting spiral NGC 5719, which is known to host two cospatial counter-rotating stellar discs .
\end{abstract}

\section{Introduction, analisys and results}

The presence of stars counter-rotating with respect to other stars and/or gas has been detected in several disc galaxies and is commonly interpreted as the end result of a retrograde acquisition of external gas and subsequent star formation (Corsini 2014). This picture can be directly tested in the NGC 5719/13 galaxy pair. NGC 5719 is an almost edge-on Sab galaxy with a prominent skewed dust lane at a distance of $23.2 \mathrm{Mpc}$.

The integral-field spectroscopic observations were carried out in service mode with the Very Large Telescope at the European Southern Observatory in Paranal. The HR blue grism covering the spectral range 4150-6200 $\AA$ and the 0.67 arcsec per fibre resolution were used. The instrumental spectral resolution was equivalent to $115 \mathrm{~km} \mathrm{~s}^{-1}$ (FWHM).

At each position in the field of view, the observed galaxy spectrum was decomposed into the contributions of the spectra of two stellar and one ionized-gas components. Therefore we measured separately the kinematics and line strengths of the Lick indices of the two stellar counter-rotating components. We also derived the kinematic of the gaseous component. Finally, we modeled the data of each stellar component with single stellar population models that account for the $[\alpha / \mathrm{Fe}]$ overabundance.

We find that the stellar counter-rotating component is younger, less rich in metals, more $\alpha$-enhanced, and less luminous with respect the main stellar body of the galaxy and it is kinematically associated with the ionized-gas disc (Coccato et al. 2011). These findings prove the scenario where gas was accreted first by NGC 5719 on to a retrograde orbit from the large reservoir available in its neighbourhoods as a result of the interaction with its companion NGC 5713, and subsequently fuelled the in situ formation of the counter-rotating stellar disc.

\section{References}

Coccato, L., Morelli, L., Corsini, E. M., et al. 2011, MNRAS, 412, L113

Corsini, E. M. 2014, Astronomical Society of the Pacific Conference Series, 486, 51 